\title{
Biplanes with flag-transitive automorphism groups of almost simple type, with exceptional socle of Lie type
}

\author{
Eugenia O'Reilly-Regueiro
}

Received: 23 November 2006 / Accepted: 14 August 2007 / Published online: 13 September 2007

(C) Springer Science+Business Media, LLC 2007

\begin{abstract}
In this paper we prove that there is no biplane admitting a flag-transitive automorphism group of almost simple type, with exceptional socle of Lie type. A biplane is a $(v, k, 2)$-symmetric design, and a flag is an incident point-block pair. A group $G$ is almost simple with socle $X$ if $X$ is the product of all the minimal normal subgroups of $G$, and $X \unlhd G \leq$ Aut $(G)$.

Throughout this work we use the classification of finite simple groups, as well as results from P.B. Kleidman's Ph.D. thesis which have not been published elsewhere.
\end{abstract}

Keywords Automorphism group · Flag-transitive $\cdot$ Primitive group $\cdot$ Symmetric design

\section{Introduction}

A biplane is a $(v, k, 2)$-symmetric design, that is, an incidence structure of $v$ points and $v$ blocks such that every point is incident with exactly $k$ blocks, and every pair of blocks is incident with exactly two points. Points and blocks are interchangeable in the previous definition, due to their dual role. A nontrivial biplane is one in which $1<k<v-1$. A flag of a biplane $D$ is an ordered pair $(p, B)$ where $p$ is a point of $D, B$ is a block of $D$, and they are incident. Hence if $G$ is an automorphism group of $D$, then $G$ is flag-transitive if it acts transitively on the flags of $D$.

The only values of $k$ for which examples of biplanes are known are $k=3,4,5,6$, 9, 11, and 13 [7, p. 76]. Due to arithmetical restrictions on the parameters, there are no examples with $k=7,8,10$, or 12 .

E. O’Reilly-Regueiro ( $\varangle)$

Instituto de Matemáticas, Universidad Nacional Autónoma de México, Circuito Exterior, Ciudad Universitaria, México, DF 04510, Mexico

e-mail: eugenia@matem.unam.mx 
For $k=3,4$, and 5 the biplanes are unique up to isomorphism [5], for $k=6$ there are exactly three non-isomorphic biplanes [11], for $k=9$ there are exactly four nonisomorphic biplanes [31], for $k=11$ there are five known biplanes [3, 9, 10], and for $k=13$ there are two known biplanes [1], in this case, it is a biplane and its dual.

In [28] it is shown that if a biplane admits an imprimitive, flag-transitive automorphism group, then it has parameters $(16,6,2)$. There are three non-isomorphic biplanes with these parameters [4], two of which admit flag-transitive automorphism groups which are imprimitive on points, (namely $2^{4} S_{4}$ and $\left(\mathbb{Z}_{2} \times \mathbb{Z}_{8}\right) S_{4}$ [28]). Therefore, if any other biplane admits a flag-transitive automorphism group $G$, then $G$ must be primitive. The O'Nan-Scott Theorem classifies primitive groups into five types [17]. It is shown in [28] that if a biplane admits a flag-transitive, primitive, automorphism group, it can only be of affine or almost simple type. The affine case was treated in [28]. The almost simple case when the socle of $G$ is an alternating or a sporadic group was treated in [29], in which it is shown that no such biplane exists. The almost simple case with classical socle was treated in [30] where it was shown that if such a biplane exists, it must have parameters $(7,4,2)$ or $(11,4,2)$ and is unique up to isomorphism. In this paper we treat the almost simple case when the socle $X$ of $G$ is an exceptional group of Lie type, and we prove that no such biplane exists, namely:

Theorem 1 (Main) There is no biplane admitting a flag-transitive, primitive almost simple automorphism group with exceptional socle of Lie type.

In [30] the proof for biplanes follows the proof given in [32] for linear spaces. The last section in [32] is an appendix on exceptional groups of Lie type, the presentation of which is also followed here.

\section{Preliminary results}

In this section we state some results that we will use in the proof of our Main Theorem.

Lemma 1 If $D$ is a (v, $k, 2)$-biplane, then $8 v-7$ is a square.

Proof The result follows from [28, Lemma 3].

Corollary 2 If $D$ is a flag-transitive $(v, k, 2)$-biplane, then $2 v<k^{2}$, and hence $2|G|<\left|G_{x}\right|^{3}$.

Proof Fix a point $x$ in the biplane. Now count flags $(p, B)$ where $p \neq x$ and $x$ is incident with the block $B$. On one hand, there are $(v-1)$ points different from $x$ and each of them is, together with $x$, in 2 blocks, so there are $2(v-1)$ such flags. On the other hand, there are $k$ blocks through $x$, and each of them has $k-1$ points different from $x$, that yields $k(k-1)$ flags. So $k(k-1)=2(v-1)$. The equality $k(k-1)=2(v-1)$ implies $k^{2}=2 v-2+k$, so clearly $2 v<k^{2}$. Since $v=\left|G: G_{x}\right|$, and $k \leq\left|G_{x}\right|$, the result follows. 
Lemma 3 (Tits Lemma) [33, 1.6] If X is a simple group of Lie type in characteristic $p$, then any proper subgroup of index prime to $p$ is contained in a parabolic subgroup of $X$.

Lemma 4 If $X$ is a simple group of Lie type in characteristic $2,\left(X ¥ A_{5}\right.$ or $\left.A_{6}\right)$, then any proper subgroup $H$ such that $[X: H]_{2} \leq 2$ is contained in a parabolic subgroup of $X$.

Proof First assume that $X=C l_{n}(q)$ is classical ( $q$ a power of 2), and take $H$ maximal in $X$. By a theorem of Aschbacher [2], $H$ is contained in a member of the collection $\mathcal{C}$ of subgroups of $\Gamma L_{n}(q)$, or in $\mathcal{S}$, that is, $H^{(\infty)}$ is quasisimple, absolutely irreducible, not realizable over any proper subfield of $\mathbb{F}_{q}$. (For a more precise description of this collection of subgroups, see [14].)

We check for every family $\mathcal{C}_{i}$ that if $H$ is contained in $C_{i}$, then $2|H|_{2}<|X|_{2}$, except when $H$ is parabolic.

Now we take $H \in \mathcal{S}$. Then by [15, Theorem 4.2], $|H|<q^{2 n+4}$, or $H$ and $X$ are as in [15, Table 4]. If $|X|_{2} \leq 2|H|_{2} \leq q^{2 n+4}$, then if $X=L_{n}^{\epsilon}(q)$ we have $n \leq 6$, and if $X=S P_{n}(q)$ or $P \Omega_{n}^{\epsilon}(q)$ then $n \leq 10$. We check the list of maximal subgroups of $X$ for $n \leq 10$ in [12, Chapter 5], and we see that no group $H$ satisfies $2|H|_{2} \leq|X|_{2}$. We then check the list of groups in [15, Table 4], and again, none of them satisfy this bound.

Finally, assume $X$ to be an exceptional group of Lie type in characteristic 2 . Then by [20], if $2|H| \geq|X|_{2}, H$ is either contained in a parabolic subgroup, or $H$ and $X$ are as in [20, Table 1]. Again, we check all the groups in [20, Table 1], and in all cases $2|H|_{2}<|X|_{2}$.

As a consequence, we have a strengthening of Corollary 2:

Corollary 5 Suppose $D$ is a biplane with a primitive, flag-transitive almost simple automorphism group $G$ with simple socle $X$ of Lie type in characteristic $p$, and the stabilizer $G_{x}$ is not a parabolic subgroup of $G$. If $p$ is odd then $p$ does not divide $k$; and if $p=2$ then 4 does not divide $k$. Hence $|G|<2\left|G_{x}\right|\left|G_{x}\right|_{p^{\prime}}^{2}$.

Proof We know from Corollary 2 that $|G|<\left|G_{x}\right|^{3}$. Now, by Lemma 3, $p$ divides $v=\left[G: G_{x}\right]$. Since $k$ divides $2(v-1)$, if $p$ is odd then $(k, p)=1$, and if $p=2$ then $(k, p) \leq 2$. Hence $k$ divides $2\left|G_{x}\right|_{p^{\prime}}$, and since $2 v<k^{2}$, we have $|G|<2\left|G_{x}\right|\left|G_{x}\right|_{p^{\prime}}^{2}$.

From the previous results we have the following lemma, which will be quite useful throughout this paper:

Lemma 6 Suppose p divides $v$, and $G_{x}$ contains a normal subgroup $H$ of Lie type in characteristic $p$ which is quasisimple and $p \nmid|Z(H)|$; then $k$ is divisible by $[H: P]$, for some parabolic subgroup $P$ of $H$.

Proof As $p$ divides $v$, and $k$ divides $2(v-1)$ we have $(k, p) \leq(2, p)$. Also, we have $k=\left[G_{x}: G_{x, B}\right]$ (where $B$ is a block incident with $x$ ), so $\left[\bar{H}: H_{B}\right]$ divides $k$, and 
therefore $\left(\left[H: H_{B}\right], p\right) \leq(2, p)$. This, and Lemmas 3 and 4 imply $H_{B}$ is contained in a parabolic subgroup $P$ of $G_{x}$, and since $P$ is maximal, we have $G_{x, B}$ is contained in $P$, so $k$ is divisible by $\left[G_{x}: P\right]$.

We will also use the following two lemmas:

Lemma 7 [18] If $X$ is a simple group of Lie type in odd characteristic, and $X$ is neither $P S L_{d}(q)$ nor $E_{6}(q)$, then the index of any parabolic subgroup is even.

Lemma 8 [22, 3.9] If $X$ is a group of Lie type in characteristic p, acting on the set of cosets of a maximal parabolic subgroup, and $X$ is not $P S L_{d}(q), P \Omega_{2 m}^{+}(q)$ (with $m$ odd), nor $E_{6}(q)$, then there is a unique subdegree which is a power of $p$.

Before stating the next result, we give the following [21]:

Definition 9 Let $H$ be a simple adjoint algebraic group over an algebraically closed field of characteristic $p>0$, and let $\sigma$ be an endomorphism of $H$ such that $X=\left(H_{\sigma}\right)^{\prime}$ is a finite simple exceptional group of Lie type over $\mathbb{F}_{q}$, where $\left(q=p^{a}\right)$. Let $G$ be a group such that $\operatorname{Soc}(G)=X$. The group $\operatorname{Aut}(X)$ is generated by $H_{\sigma}$, together with field and graph automorphisms. If $D$ is a $\sigma$-stable closed connected reductive subgroup of $H$ containing a maximal torus $T$ of $H$, and $M=N_{G}(D)$, then we call $M$ a subgroup of maximal rank in $G$.

We now have the following theorem and table [24, Theorem 2, Table III]:

Theorem 10 If $X$ is a finite simple exceptional group of Lie type such that $X \leq G \leq$ $\operatorname{Aut}(X)$, and $G_{X}$ is a maximal subgroup of $G$ such that $X_{0}=\operatorname{Soc}\left(G_{X}\right)$ is not simple, then one of the following holds:

(1) $G_{x}$ is parabolic.

(2) $G_{x}$ is of maximal rank.

(3) $G_{x}=N_{G}(E)$, where $E$ is an elementary Abelian group given in $[6$, Theorem $1(I I)$.$] .$

(4) $X=E_{8}(q),(p>5)$, and $X_{0}$ is either $A_{5} \times A_{6}$ or $A_{5} \times L_{2}(q)$.

(5) $X_{0}$ is as in Table 1.

We will also use the following theorem [23, Theorem 3]:

Theorem 11 Let $X$ be a finite simple exceptional group of Lie type, with $X \leq G \leq$ $\operatorname{Aut}(X)$. Assume $G_{x}$ is a maximal subgroup of $G$, and $\operatorname{Soc}\left(G_{x}\right)=X_{0}(q)$ is a simple group of Lie type over $\mathbb{F}_{q}(q>2)$ such that $\frac{1}{2} \operatorname{rk}(X)<\operatorname{rk}\left(X_{0}\right)$. Then one of the following holds:

(1) $G_{x}$ is a subgroup of maximal rank.

(2) $X_{0}$ is a subfield or twisted subgroup.

(3) $X=E_{6}(q)$ and $X_{0}=C_{4}(q)$ ( $q$ odd) or $F_{4}(q)$. 


\section{Table 1}

\begin{tabular}{ll}
\hline$X$ & $X_{0}$ \\
\hline$F_{4}(q)$ & $L_{2}(q) \times G_{2}(q)(p>2, q>3)$ \\
$E_{6}^{\epsilon}(q)$ & $L_{3}(q) \times G_{2}(q), U_{3}(q) \times G_{2}(q)(q>2)$ \\
$E_{7}(q)$ & $L_{2}(q) \times L_{2}(q)(p>3), L_{2}(q) \times G_{2}(q)(p>2, q>3)$ \\
& $L_{2}(q) \times F_{4}(q)(q>3), G_{2}(q) \times P S p_{6}(q)$ \\
& $L_{2}(q) \times L_{3}^{\epsilon}(q)(p>3), G_{2}(q) \times F_{4}(q)$ \\
$E_{8}(q)$ & $L_{2}(q) \times G_{2}(q) \times G_{2}(q)(p>2, q>3), L_{2}(q) \times G_{2}\left(q^{2}\right)(p>2, q>3)$ \\
\end{tabular}

Finally, we will use the following theorem [26, Theorem 1.2]:

Theorem 12 Let $X$ be a finite exceptional group of Lie type such that $X \leq G \leq$ $\operatorname{Aut}(X)$, and $G_{X}$ a maximal subgroup of $G$ with socle $X_{0}=X_{0}(q)$ a simple group of Lie type in characteristic $p$. Then if $\operatorname{rk}\left(X_{0}\right) \leq \frac{1}{2} \mathrm{rk}(X)$, we have the following bounds:

(1) If $X=F_{4}(q)$ then $\left|G_{x}\right|<q^{20} .4 \log _{p}(q)$,

(2) If $X=E_{6}^{\epsilon}$ then $\left|G_{x}\right|<q^{28} .4 \log _{p}(q)$,

(3) If $X=E_{7}(q)$ then $\left|G_{x}\right|<q^{30} .4 \log _{p}(q)$, and

(4) If $X=E_{8}(q)$ then $\left|G_{x}\right|<q^{56} .12 \log _{p}(q)$.

In all cases, $\left|G_{x}\right|<|G|^{\frac{5}{13}} \cdot 5 \log _{p}(q)$.

\section{Proof of our main theorem}

Lemma 13 The group $X$ is not a Suzuki group ${ }^{2} B_{2}(q)$, with $q=2^{2 e+1}$.

Proof Suppose that the socle $X$ is a Suzuki group ${ }^{2} B_{2}(q)$, with $q=2^{2 e+1}$. Then $|G|=f|X|=f\left(q^{2}+1\right) q^{2}(q-1)$, where $f \mid(2 e+1)$, and so the order of any point stabilizer $G_{x}$ is one of the following [34]:

(1) $f q^{2}(q-1)$

(2) $4 f(q+\sqrt{2 q}+1)$

(3) $4 f(q-\sqrt{2 q}+1)$

(4) $f\left(q_{0}^{2}+1\right) q_{0}^{2}\left(q_{0}-1\right)$, where $8 \leq q_{0}^{m}=q$, with $m \geq 3$.

Case (1) Here $v=\left(q^{2}+1\right)$, so from $k(k-1)=2(v-1)$ we obtain $k(k-1)=2 q^{2}$, a power of 2 , which is a contradiction.

Cases (2) and (3) From the inequality $|G|<\left|G_{x}\right|^{3}$, we have

$$
\begin{aligned}
f \cdot \frac{7}{8} q^{5} & <f\left(q^{2}+1\right) q^{2}(q-1)<4^{4} f^{3}(q \pm \sqrt{2 q}+1)^{3} \\
& <4^{4} f^{3}(2 q+1)^{3} \leq 4^{4}\left(\frac{17}{8} f q\right)^{3},
\end{aligned}
$$


so

$$
q^{2}<\frac{4^{4} \cdot(17)^{3} \cdot f^{2}}{8^{2} \cdot 7}<2808 f^{2}
$$

hence $q \leq 128$.

First assume $q=128$. Then $v=58781696$ and 75427840 and $\left|G_{x}\right|=4060$ and 3164 in cases (2) and (3) respectively. We know $k$ divides $2\left(\left|G_{x}\right|, v-1\right)$, but here $\left(\left|G_{x}\right|, v-1\right)=1015$ in case (2), and 113 in case (3). In both cases $k^{2}<v$, which is a contradiction.

Next assume $q=32$. Then $v=198400$ and 325376 in cases (2), and (3) respectively. In case $(2),(|G-x|, v-1)=41$, and in case $(3),\left(\left|G_{x}\right|, v-1\right)=25$ or 125 , depending on whether $f=1$ or 5 . In all cases we see $k^{2}<v$, a contradiction.

Finally assume $q=8$. Then $v=560$ and 1456, and $\left(\left|G_{x}\right|, v-1\right)=13$ and $5 f$ in cases (2) and (3) respectively, therefore $k$ is again too small.

Case (4) Here $\left|G_{x}\right|=f\left(q_{0}^{2}+1\right) q_{0}^{2}\left(q_{0}-1\right)$, so $q_{0}$ divides $v$ and hence $q_{0}$ and $v-1$ are relatively prime, so from $|G|<2\left|G_{x}\right|\left|G_{x}\right|_{p^{\prime}}^{2}$ we obtain:

$$
\left(q_{0}^{2 m}+1\right) q_{0}^{2 m}\left(q_{0}^{m}-1\right)<4 f^{2}\left(q_{0}^{2}+1\right)^{3} q_{0}^{2}\left(q_{0}-1\right)^{3} .
$$

Now, $q_{0}^{5 m-1}<\left(q_{0}^{2 m}+1\right) q_{0}^{2 m}\left(q_{0}^{m}-1\right)$, and also

$$
4 f^{2}\left(q_{0}^{2}+1\right)^{3} q_{0}^{2}\left(q_{0}-1\right)^{3}=4 f^{2} q_{0}^{2}\left(q_{0}^{3}-q_{0}^{2}+q_{0}-1\right)^{3}<f^{2} q_{0}^{13},
$$

so

$$
q_{0}^{5 m-1}<f^{2} q_{0}^{13}<q_{0}^{13+m}
$$

Therefore $5 m-1<13+m$, which forces $m=3$. Then

$$
v=\left(q_{0}^{4}-q_{0}^{2}+1\right) q_{0}^{4}\left(q_{0}^{2}+q_{0}+1\right)
$$

and so $k \leq 2\left(\left|G_{x}\right|, v-1\right) \leq 2 f q_{0}^{3}<2 q_{0}^{\frac{9}{2}}$. The inequality $v<k^{2}$ forces $q_{0}=2$, and so $q=8$. Then $v=1456$, and $\left|G_{x}\right|=20 f$, with $f=1$ or 3 . Hence $\left(\left|G_{x}\right|, v-1\right)=5 f$, and therefore $k^{2}<v$, which is a contradiction.

This completes the proof of Lemma 13.

Lemma 14 The point stabilizer $G_{x}$ is not a parabolic subgroup of $G$.

Proof First assume $X \neq E_{6}(q)$. Then by Lemma 8 there is a unique subdegree which is a power of $p$. Therefore $k$ divides twice a power of $p$, but it also divides $2(v-1)$, so it is too small.

Now assume $X=E_{6}(q)$. If $G$ contains a graph automorphism or $G_{x}=P_{i}$ with $i=2$ or 4 , then there is a unique subdegree which is a power of $p$ and again $k$ is too small. If $G_{x}=P_{3}$, the $A_{1} A_{4}$ type parabolic, then

$$
v=\frac{\left(q^{3}+1\right)\left(q^{4}+1\right)\left(q^{12}-1\right)\left(q^{9}-1\right)}{\left(q^{2}-1\right)(q-1)} .
$$


Since $k$ divides $2\left(\left|G_{x}\right|, v-1\right)$, then $k$ divides $2 q\left(q^{5}-1\right)(q-1)^{5} \log _{p} q$, and hence $k^{2}<v$, which is a contradiction. If $G_{x}=P_{1}$, then

$$
v=\frac{\left(q^{12}-1\right)\left(q^{9}-1\right)}{\left(q^{4}-1\right)(q-1)},
$$

and the nontrivial subdegrees are (see [19]): $\frac{q\left(q^{8}-1\right)\left(q^{3}+1\right)}{(q-1)}$, and $\frac{q^{8}\left(q^{5}-1\right)\left(q^{4}+1\right)}{(q-1)}$. The fact that $k$ divides twice the highest common factor of these forces $k^{2}<v$, again, a contradiction.

This completes the proof of Lemma 14.

Lemma 15 The group $X$ is not a Chevalley group $G_{2}(q)$.

Proof Assume $X=G_{2}(q)$, with $q>2$ since $G_{2}(q)^{\prime}=U_{3}(3)$. The list of maximal subgroups of $G_{2}(q)$ with $q$ odd can be found in [13], and in [8] for $q$ even.

First consider the case where $X \cap G_{x}=S L_{3}^{\epsilon}(q)$.2. Here

$$
v=\frac{q^{3}\left(q^{3}+\epsilon\right)}{2} .
$$

From the factorization $\Omega_{7}(q)=G_{2}(q) N_{1}^{\epsilon}$, ([16]), it follows that the suborbits of $\Omega_{7}(q)$ are unions of $G_{2}$-suborbits, and so $k$ divides each of the $\Omega_{7}$-subdegrees. Now $q$ cannot be odd, since this is ruled out by the first case with $i=1$ in the section of orthogonal groups of odd dimension in [30]. For $q$ even, the subdegrees for $\operatorname{Sp}_{6}(q)$, given in the last case of the section on symplectic groups in [30] are $\left(q^{3}-\epsilon\right)\left(q^{4}+\epsilon\right)$ and $\frac{(q-2) q^{2}\left(q^{3}-\epsilon\right)}{2}$. This implies that $k$ divides $2\left(q^{3}-\epsilon\right)\left(q-2, q^{2}+\epsilon\right)$, and since $v<k^{2}$ then $\epsilon=-1$, and so

$$
v=\frac{q^{3}\left(q^{3}-1\right)}{2} .
$$

So $k$ divides $2\left(q^{3}+1\right)\left(q-2, q^{2}-1\right) \leq 6\left(q^{3}+1\right)$, and $k(k-1)=2(v-1)=\left(q^{3}+1\right)$ $\left(q^{3}-2\right)$. This is impossible.

If $X \cap G_{x}=G_{2}\left(q_{0}\right)<G_{2}(q)$ or ${ }^{2} G_{2}(q)<G_{2}(q)$ then $p$ does not divide $\left[G_{x}: G_{x B}\right]$, so by Lemma $6, k$ is divisible by the index of a parabolic subgroup of $G_{x}$ which is $\frac{q_{0}^{6}-1}{q_{0}-1}$ in the case of $G_{2}\left(q_{0}\right)$, or $q^{3}+1$ in the case of ${ }^{2} G_{2}(q)$. But this is not so since $k$ also divides $2\left(v-1,\left|G_{x}\right|\right)$.

If $G_{x}=N_{G}\left(S L_{2}(q) \circ S L_{2}(q)\right)$, then

$$
v=\frac{q^{4}\left(q^{6}-1\right)}{q^{2}-1} .
$$

Now $k$ divides $2\left(q^{2}-1\right)^{2} \log _{p} q$ but $\left(q^{2}-1, v-1\right) \leq 2$, so $k$ is too small.

If $X \cap G_{x}=J_{2}<G_{2}(4)$ then $v=416$. But $k$ divides $2\left(\left|G_{x}\right|, 415\right)$, which is too small. 
Now suppose $X \cap G_{x}=G_{2}(2)$, with $p=q \geq 5$. Then the inequality $v<k^{2}$ forces $q=5$ or 7 . In both cases $\left(v-1,\left|G_{X}\right|\right)$ is too small.

If $X \cap G_{x}=P G L_{2}(q)$, or $L_{2}(8)$, then the inequality $|G|<\left|G_{x}\right|^{3}$ is not satisfied.

Next consider $X \cap G_{X}=L_{2}(13)$. Then the inequality $|G|<\left|G_{X}\right|^{3}$ forces $q \leq 5$. If $q=5$ then $v=2^{3} \cdot 3^{2} \cdot 5^{6} \cdot 13 \cdot 31$, so $\left(v-1,\left|G_{x}\right|\right) \leq 7$, hence $k$ is too small. If $q=3$ then $v=2^{3} \cdot 3^{5}$, and $k$ divides $2\left(v-1,\left|G_{x}\right|\right) \leq 2 \cdot 7 \cdot 13$, this does not satisfy the equation $k(k-1)=2(v-1)$.

Finally, if $X \cap G_{x}=J_{1}$ with $q=11$ then the inequality $v<k^{2}$ cannot be satisfied.

There is no other maximal subgroup $G_{X}$ satisfying the inequality $|G|<\left|G_{x}\right|$.

This completes the proof of Lemma 15.

Lemma 16 The group $X$ is not a Ree group ${ }^{2} G_{2}(q),(q>3)$.

Proof Suppose $X={ }^{2} G_{2}(q)$, with $q=3^{2 e+1}>3$. A complete list of maximal subgroups of $G$ can be found in [13, p. 61]. First suppose $G_{X} \cap X=2 \times S L_{2}(q)$. Then

$$
v=\frac{q^{2}\left(q^{2}-q+1\right)}{2},
$$

so $2(v-1)=q^{4}-q^{3}+q^{2}-2$, and $k$ divides $2\left(\left|G_{x}\right|, v-1\right)$. But $\left(q\left(q^{2}-1\right), q^{4}-\right.$ $\left.q^{3}+q^{2}-1\right)=q-1$, which is too small.

The groups $X \cap G_{x}=N_{X}\left(S_{2}\right)$, (where $S_{2}$ is a Sylow 2-subgroup of $X$ of order 8), of order $2^{3} \cdot 3 \cdot 7$ and $L_{2}(8)$ are not allowed since $|G|<\left|G_{x}\right|^{3}$ forces $q=3$.

If $X \cap G_{x}={ }^{2} G_{2}\left(q_{0}\right)$, with $q_{0}^{m}=q$ and $m$ prime, then

$$
\begin{aligned}
v= & q_{0}^{3(m-1)}\left(q_{0}^{3(m-1)}-q_{0}^{3(m-2)}+\ldots+(-1)^{m} q_{0}^{3}+(-1)^{m-1}\right) \\
& \times\left(q_{0}^{m-1}+q_{0}^{m-2}+\ldots+1\right) .
\end{aligned}
$$

Now $k$ divides $2 m q_{0}^{3}\left(q_{0}^{3}+1\right)\left(q_{0}-1\right)$, but since $q_{0}$ and $v-1$ are relatively prime, $q_{0}$ does not divide $k$, so in fact $k \leq 2 m\left(q_{0}^{3}+1\right)\left(q_{0}-1\right)$, and the inequality $v<k^{2}$ forces $m=2$, which is a contradiction. fied.

If $X \cap G_{x}=\mathbb{Z}_{q \pm \sqrt{3 q}+1}: \mathbb{Z}_{6}$, since $q \geq 27$ the inequality $|G|<\left|G_{x}\right|^{3}$ is not satis-

Finally, if $X \cap G_{x}=\left(2^{2} \times D_{\left(\frac{1}{2}\right)(q+1)}\right): 3$, since $q \geq 27$ then the inequality $|G|<$ $\left|G_{x}\right|^{3}$ is not satisfied.

This completes the proof of Lemma 16.

Lemma 17 The group $X$ is not a Ree group ${ }^{2} F_{4}(q)$.

Proof Suppose $X={ }^{2} F_{4}(q)$. Then from [27] we see there are no maximal subgroups $G_{x}$ that are not parabolic satisfying the inequality $|G|<2\left|G_{x}\right|\left|G_{x}\right|_{2^{\prime}}^{2}$, except for the case $q=2$. In this case $G_{x} \cap X=L_{3}(3) .2$ or $L_{2}(25)$. In both cases, since $k$ must divide $2\left(v-1,\left|G_{x}\right|\right)$ it is too small. 
Lemma 18 The group $X$ is not ${ }^{3} D_{4}(q)$.

Proof Suppose $X={ }^{3} D_{4}(q)$. If $X \cap G_{x}=G_{2}(q)$ or $S L_{2}\left(q^{3}\right) \circ S L_{2}(q) .(2, q-1)$ then $v=q^{e}\left(q^{8}+q^{4}+1\right)$, where $e=6$ or 8 respectively. By Lemma $6, k$ is divisible by $q+1$, which forces $q=3$ (since $q+1$ also divides $2(v-1)$ ), but then in neither case is $8 v-7$ a square.

If $X \cap G_{x}=P G L_{3}^{\epsilon}(q)$ then the inequality $|G|<\left|G_{x}\right|^{3}$ is not satisfied.

Lemma 19 The group $X$ is not $F_{4}(q)$.

Proof Suppose $X=F_{4}(q)$. First assume that $X_{0}=\operatorname{Soc}\left(X \cap G_{X}\right)$ is not simple. Then by Theorem 10 and Table $1, G_{X} \cap X$ is one of the following,

(1) Parabolic.

(2) Of maximal rank.

(3) $3^{3} \cdot S L_{3}(3)$.

or $X_{0}=L_{2}(q) \times G_{2}(q)(p>2, q>3)$.

The parabolic subgroups have been ruled out by Lemma 14 .

The possibilities for the second case are given in [21, Table 5.1]. We check that in every case there is a large power of $q$ dividing $v$, and since $(k, v) \leq 2$, then $q \neq 2$ does not divide $k$. If $q=2$, then 4 does not divide $k$. Therefore $k$ divides $2\left(\left|G_{x}\right|, v-1\right)$, and in each case $\left(\left|G_{x}\right|_{p^{\prime}}, v-1\right)$ is too small for $k$ to satisfy $k^{2}>v$.

The local subgroup is too small to satisfy the bound $\left|G_{x}\right|^{3}>|G|$.

Finally, $\left|L_{2}(q) \times G_{2}(q)\right| \leq q^{7}\left(q^{2}-1\right)^{2}\left(q^{6}-1\right)<\left|F_{4}(q)\right|^{\frac{1}{3}}$. Therefore $X_{0}$ is simple.

First suppose $X_{0} \notin \operatorname{Lie}(p)$. Then by [25, Table 1], it is one of the following:

$A_{7}, A_{8}, A_{9}, A_{10}, L_{2}(17), L_{2}(25), L_{2}(27), L_{3}(3), U_{4}(2), S p_{6}(2), \Omega_{8}^{+}(2),{ }^{3} D_{4}(2)$, $J_{2}, A_{11}(p=11), L_{3}(4)(p=3), L_{4}(3)(p=2),{ }^{2} B_{2}(8)(p=5), M_{11}(p=11)$.

The only possibilities for $X_{0}$ that could satisfy the bound $\left|G_{x}\right|^{3}>|G|$ are $A_{9}, A_{10}(q=2), S p_{6}(2)(q=2), \Omega_{8}^{+}(2)(q=2,3),{ }^{3} D_{4}(2)(q=3), J_{2}(q=2)$, and $L_{4}(3)(q=2)$. However, since $k$ divides $2\left(\left|G_{x}\right|, v-1\right)$, in all these cases $k^{2}<v$.

Now assume $X_{0} \in \operatorname{Lie}(p)$. First consider the case $\operatorname{rk}\left(X_{0}\right)>\frac{1}{2} \operatorname{rk}(G)$, where $X_{0}=$ $X_{0}(r)$. If $r>2$, then by Theorem 11 it is a subfield subgroup. We have seen earlier that the only subgroups which could satisfy the bound $\left|G_{x}\right|^{3}>|G|$ are $F_{4}\left(q^{\frac{1}{2}}\right)$ and $F_{4}\left(q^{\frac{1}{3}}\right)$. If $q_{0}=q^{\frac{1}{2}}$, then

$$
v=q^{12}\left(q^{6}+1\right)\left(q^{4}+1\right)\left(q^{3}+1\right)(q+1)>q^{26} .
$$

Now $k$ divides $2 F_{4}\left(q^{\frac{1}{2}}\right)$, and $(k, v) \leq 2$. Since $(q, k) \leq 2$, then $k$ divides

$$
2\left(2\left(q^{6}-1\right)\left(q^{4}-1\right)\left(q^{3}-1\right)(q-1), v-1\right)<q^{13},
$$

so $k^{2}<v$, a contradiction.

If $q_{0}=q^{\frac{1}{3}}$, then

$$
v=\frac{q^{16}\left(q^{12}-1\right)\left(q^{4}+1\right)\left(q^{6}-1\right)}{\left(q^{\frac{8}{3}}-1\right)\left(q^{\frac{2}{3}}-1\right)}
$$


but $k<q^{10}$, so $k^{2}<v$, which is a contradiction.

If $r=2$, then the subgroups $X_{0}(2)$ with $\operatorname{rk}\left(X_{0}\right)>\frac{1}{2} \operatorname{rk}(G)$ that satisfy the bound $\left|G_{x}\right|^{3}>|G|$ are $A_{4}^{\epsilon}(2), B_{3}(2), B_{4}(2), C_{3}(2), C_{4}(2)$, and $D_{4}^{\epsilon}(2)$. Again, in all cases the fact that $k$ divides $2\left(\left|G_{x}\right|, v-1\right)$ forces $k^{2}<v$, a contradiction.

Now consider the case $\operatorname{rk}\left(X_{0}\right) \leq \frac{1}{2} \operatorname{rk}(G)$. Theorem 12 implies $\left|G_{x}\right|<q^{20} .4 \log _{p} q$. Looking at the orders of groups of Lie type, we see that if $\left|G_{x}\right|<q^{20} .4 \log _{p} q$, then $\left|G_{x}\right|_{p^{\prime}}<q^{12}$, so $2\left|G_{x}\right|\left|G_{x}\right|_{p^{\prime}}^{2}<|G|$, contrary to Corollary 5.

This completes the proof of Lemma 19.

Lemma 20 The group $X$ is not $E_{6}^{\epsilon}(q)$.

Proof Suppose $X=E_{6}^{\epsilon}(q)$. As in the previous lemma, assume first that $X_{0}$ is not simple. Then Theorem 10 implies $G_{X} \cap X$ is one of the following,

(1) Parabolic.

(2) Of maximal rank.

(3) $3^{6} \cdot S L_{3}(3)$.

or $X_{0}=L_{3}(q) \times G_{2}(q), U_{3}(q) \times G_{2}(q)(q>2)$.

The first case was ruled out in Lemma 14.

The possibilities for the second case are given in [21, Table 5.1]. In some cases $\left|G_{x}\right|^{3}<|G|$, and in each of the remaining cases, calculating $2\left(\left|G_{x}\right|, v-1\right)$ we obtain $k^{2}<v$.

The local subgroup for the third case is too small.

Finally, the orders of the groups in the last case are less than $q^{17}<\left|E_{6}^{\epsilon}\right|^{\frac{1}{3}}$.

Now assume $X_{0}$ is simple. If $X_{0} \notin \operatorname{Lie}(p)$, then we find the possibilities in [25, Table 1]. However, the only two cases which satisfy Corollary 2 have order that does not divide $\left|E_{6}^{\epsilon}\right|$. Hence $X_{0}=X_{0}(r) \in \operatorname{Lie}(p)$.

If $\operatorname{rk}\left(X_{0}\right)>\frac{1}{2} \operatorname{rk}(G)$, then when $r>2$ by Theorem 11 the only possibilities are $E_{6}^{\epsilon}\left(q^{\frac{1}{s}}\right)$ with $s=2$ or $3, C_{4}(q)$, and $F_{4}(q)$. In all cases $k$ is too small. When $q=2$ then the possibilities satisfying $\left|G_{x}\right|^{3}>|G|$ with order dividing $E_{6}^{\epsilon}(2)$ are $A_{5}^{\epsilon}(2)$, $B_{4}(2), C_{4}(2), D_{4}^{\epsilon}(2)$, and $D_{5}^{\epsilon}(2)$. However since $k$ divides $2\left(\left|G_{x}\right|, v-1\right)$, in all cases $k^{2}<v$, a contradiction.

If $\operatorname{rk}\left(X_{0}\right) \leq \frac{1}{2} \operatorname{rk}(G)$, then Theorem 12 implies $\left|G_{x}\right|<q^{28} .4 \log _{p} q$. Looking at the $p-$ and $p^{\prime}-$ parts of the orders of the possible subgroups, we see that the $p^{\prime}$-part is always less than $q^{17}$. Hence $\left|G_{x}\right|_{p^{\prime}}<q^{17}$, so $2\left|G_{x}\right|\left|G_{x}\right|_{p^{\prime}}^{2}<|G|$, contradicting Corollary 5.

This completes the proof of Lemma 20.

Lemma 21 The group $X$ is not $E_{7}(q)$.

Proof Suppose $X=E_{7}(q)$. First assume $X_{0}$ is not simple. Then by Theorem 10 , $G_{X} \cap X$ is one of the following,

(1) Parabolic. 
(2) Of maximal rank.

(3) $2^{2} \cdot S_{3}$.

or $X_{0}=L_{2}(q) \times L_{2}(q)(p>3), \quad L_{2}(q) \times G_{2}(q)(p>2, q>3), \quad L_{2}(q) \times$ $F_{4}(q)(q>3)$, or $G_{2}(q) \times P \operatorname{Sp}_{6}(q)$.

The parabolic subgroups have been ruled out in Lemma 14. The subgroups of maximal rank can be found in [21, Table 5.1]. Of these, the only ones with order greater than $\left|E_{7}(q)\right|^{\frac{1}{3}}$ are $d .\left(L_{2}(q) \times P \Omega_{12}^{+}(q)\right) . d$ and $f . L_{8}^{\epsilon}(q) \cdot g .\left(2 \times\left(\frac{2}{f}\right)\right)$, where $d=(2, q-1), f=\left(4, \frac{q-\epsilon}{d}\right)$, and $g=\left(8, \frac{q-\epsilon}{d}\right)$. However in both cases the fact that $(k, v) \leq 2$ forces $k^{2}<v$, a contradiction.

The local subgroup is too small to satisfy $\left|G_{x}\right|^{3}>|G|$.

In the last case, the only group that is not too small to satisfy $\left|G_{x}\right|^{3}>|G|$ is $L_{2}(q) \times F_{4}(q)$, but here $q^{38}$ divides $v$, and since $(v, k) \leq 2$, then $k^{2}<v$. So $X_{0}$ is simple.

First assume $X_{0} \notin \operatorname{Lie}(p)$. Then by [25, Table 1], the possibilities are $A_{14}(p=7)$, $M_{22}(p=5), R u(p=5)$, and $H S(p=5)$. None of these groups satisfy Corollary 2.

Now assume $X_{0}=X_{0}(r) \in \operatorname{Lie}(p)$. If $\operatorname{rk}\left(X_{0}\right) \leq \frac{1}{2} \operatorname{rk}(G)$, then by Theorem 12, $\left|G_{x}\right|^{3}<|G|$, which is a contradiction.

If $\operatorname{rk}\left(X_{0}\right)>\frac{1}{2} \operatorname{rk}(G)$ then if $r>2$ Theorem 11 implies $X \cap G_{x}=E_{7}\left(q^{\frac{1}{s}}\right)$, with $s=$ 2 or 3 . However in both cases $(v, k) \leq 2$ forces $k^{2}<v$, a contradiction. If $r=2$ then the possible subgroups satisfying the bound $\left|G_{x}\right|^{3}>|G|$ and having order dividing $\left|E_{7}(2)\right|$ are $A_{6}^{\epsilon}(2), A_{7}^{\epsilon}(2), B_{5}(2), C_{5}(2), D_{5}^{\epsilon}(2)$, and $D_{6}^{\epsilon}(2)$. However in all of these cases $(v, k) \leq 2$ forces $k^{2}<v$.

Lemma 22 The group $X$ is not $E_{8}(q)$.

Proof Suppose $X=E_{8}(q)$. First suppose that $X_{0}$ is not simple. Then by Theorem 10, $G_{X} \cap X$ is one of the following,

(1) Parabolic.

(2) Of maximal rank.

(3) $\left(2^{15}\right) \cdot L_{5}(2)(q$ odd $)$ or $5^{3} \cdot S L_{3}(5)\left(5 \mid q^{2}-1\right)$.

(4) $G_{x} \cap X=\left(A_{5} \times A_{6}\right) \cdot 2^{2}$.

or $X_{0}=L_{2}(q) \times L_{3}^{\epsilon}(q)(p>3), G_{2}(q) \times F_{4}(q), L_{2}(q) \times G_{2}(q) \times G_{2}(q)(p>2, q>$ $3)$, or $L_{2}(q) \times G_{2}\left(q^{2}\right)(p>2, q>3)$.

We know from Lemma 14 that the first case does not hold.

From [21, Table 5.1] the only subgroups of maximal rank such that $\left|G_{x}\right|^{3} \geq|G|$ are $d . P \Omega_{16}^{+}(q) . d, d .\left(L_{2}(q) \times E_{7}(q)\right) . d, f . L_{9}^{\epsilon}(q) . e .2$, and $e .\left(L_{3}^{\epsilon}(q) \times E_{6}^{\epsilon}(q)\right) . e .2$, (where $d=(2, q-1), e=(3, q-\epsilon)$, and $\left.f=\frac{(9, q-\epsilon)}{e}\right)$. In all cases, $(k, v) \leq 2$ implies $k^{2}<v$, which is a contradiction.

In all other cases, for all possible groups we have that $\left|G_{x}\right|^{3}<|G|$, a contradiction. Hence $X_{0}$ is simple.

First consider the case $X_{0} \notin \operatorname{Lie}(p)$. Then by [25, Table 1] the possibilities are $A l t_{14}, A l t_{15}, A l t_{16}, A l t_{17}, A l t_{18}(p=3), L_{2}(16), L_{2}(31), L_{2}(32), L_{2}(41)$, 
$L_{2}(49), L_{2}(61), L_{3}(5), L_{4}(5)(p=2), P S p_{4}(5), G_{2}(3),{ }^{2} B_{2}(8),{ }^{2} B_{2}(32)(p=5)$, and $T h(p=3)$. In every case the inequality $\left|G_{X}\right|^{3}>|G|$ is not satisfied.

Now consider the case $X_{0} \in \operatorname{Lie}(p)$. If $\operatorname{rk}\left(X_{0}\right) \leq \frac{1}{2} \operatorname{rk}(G)$, then by Theorem 12 we have $\left|G_{x}\right|^{3} \geq|G|$, which is a contradiction.

So $\operatorname{rk}\left(X_{0}\right)>\frac{1}{2} \operatorname{rk}(G)$. If $r>2$, then by Theorem $11, G_{x} \cap X$ is a subfield subgroup. The only cases in which $\left|G_{x}\right|^{3}>|G|$ can be satisfied are when $q=q_{0}^{2}$ or $q=q_{0}^{3}$, but in all cases since $(v, k) \leq 2$ then $k$ is too small.

If $r=2$, then $\operatorname{rk}\left(X_{0}\right) \geq 5$. The groups for which $|G|<\left|G_{x}\right|^{3}$ are $A_{8}^{\epsilon}(2), B_{8}(2)$, $B_{7}(2), C_{8}(2), C_{7}(2), D_{8}^{\epsilon}(2)$, and $D_{7}^{\epsilon}(2)$. However, in all cases $(v, k) \leq 2$ forces $k^{2}<v$, which is a contradiction.

This completes the proof of Lemma 22, completing thus the proof of our Main Theorem. As a consequence of this and the results in $[29,30]$ we have the following:

Theorem 23 If $D$ is a biplane with a primitive, flag-transitive automorphism group of almost simple type, then $D$ has parameters either $(7,4,2)$, or $(11,5,2)$, and is unique up to isomorphism.

Acknowledgements The results in the present paper were obtained during the course of my Ph.D. under the supervision of Martin W. Liebeck, with a grant from the Dirección General de Asuntos del Personal Académico, Universidad Nacional Autónoma de México. I am very grateful to Martin for his guidance and help. I would also like to thank Jan Saxl for allowing me to view his notes before [32] was published, and Sasha Ivanov for providing me with these notes.

I would also like to thank the referees, who provided me with helpful suggestions.

\section{References}

1. Aschbacher, M.: On collineation groups of symmetric block designs. J. Comb. Theory 11, 272-281 (1971)

2. Aschbacher, M.: On the maximal subgroups of the finite classical groups. Invent. Math. 76, 469-514 (1984)

3. Assmus, E.F. Jr., Mezzaroba, J.A., Salwach, C.J.: Planes and biplanes. In: Proceedings of the 1976 Berlin Combinatorics Conference, Vancerredle (1977)

4. Assmus, E.F. Jr., Salwach, C.J.: The (16,6,2) designs. Int. J. Math. Math. Sci. 2(2), 261-281 (1979)

5. Cameron, P.J.: Biplanes. Math. Z. 131, 85-101 (1973)

6. Cohen, A.M., Liebeck, M.W., Saxl, J., Seitz, G.M.: The local maximal subgroups of exceptional groups of Lie type, finite and algebraic. Proc. Lond. Math. Soc. (3) 64, 21-48 (1992)

7. Colburn, C.J., Dinitz, J.H.: The CRC Handbook of Combinatorial Designs. CRC Press, Boca Raton (1996)

8. Cooperstein, B.N.: Minimal degree for a permutation representation of a classical group. Isr. J. Math. 30, 213-235 (1978)

9. Denniston, R.H.F.: On biplanes with 56 points. Ars. Comb. 9, 167-179 (1980)

10. Hall, M. Jr., Lane, R., Wales, D.: Designs derived from permutation groups. J. Comb. Theory 8, 12-22 (1970)

11. Hussain, Q.M.: On the totality of the solutions for the symmetrical incomplete block designs $\lambda=2$, $k=5$ or 6 . Sankhya 7, 204-208 (1945)

12. Kleidman, P.B.: The subgroup structure of some finite simple groups. PhD thesis, University of Cambridge (1987)

13. Kleidman, P.B.: The maximal subgroups of the Chevalley groups $G_{2}(q)$ with $q$ odd, the Ree groups ${ }^{2} G_{2}(q)$, and their automorphism groups. J. Algebra 117, 30-71 (1998)

14. Kleidman, P.B., Liebeck, M.W.: The Subgroup Structure of the Finite Classical Groups. London Mathematical Society Lecture Note Series, vol. 129. Cambridge University Press, Cambridge (1990) 
15. Liebeck, M.W.: On the orders of maximal subgroups of the finite classical groups. Proc. Lond. Math. Soc. 50, 426-446 (1985)

16. Liebeck, M.W., Praeger, C.E., Saxl, J.: The maximal factorizations of the finite simple groups and their automorphism groups. Mem. Am. Math. Soc. 86(432), 1-151 (1990)

17. Liebeck, M.W., Praeger, C.E., Saxl, J.: On the O’Nan-Scott theorem for finite primitive permutation groups. J. Austral. Math. Soc. (Ser. A) 44, 389-396 (1988)

18. Liebeck, M.W., Saxl, J.: The primitive permutation groups of odd degree. J. Lond. Math. Soc. 31, 250-264 (1985)

19. Liebeck, M.W., Saxl, J.: The finite primitive permutation groups of rank three. Bull. Lond. Math. Soc. 18, 165-172 (1986)

20. Liebeck, M.W., Saxl, J.: On the orders of maximal subgroups of the finite exceptional groups of Lie type. Proc. Lond. Math. Soc. 55, 299-330 (1987)

21. Liebeck, M.W., Saxl, J., Seitz, G.M.: Subgroups of maximal rank in finite exceptional groups of Lie type. Proc. Lond. Math. Soc. 65, 297-325 (1992)

22. Liebeck, M.W., Saxl, J., Seitz, G.M.: On the overgroups of irreducible subgroups of the finite classical groups. Proc. Lond. Math. Soc. 55, 507-537 (1987)

23. Liebeck, M.W., Saxl, J., Testerman, D.M.: Simple subgroups of large rank in groups of Lie type. Proc. Lond. Math. Soc. (3) 72, 425-457 (1996)

24. Liebeck, M.W., Seitz, G.M.: Maximal subgroups of exceptional groups of Lie type, finite and algebraic. Geom. Dedic. 35, 353-387 (1990)

25. Liebeck, M.W., Seitz, G.M.: On finite subgroups of exceptional algebraic groups. J. Reine Angew. Math. 515, 25-72 (1999)

26. Liebeck, M.W., Shalev, A.: The probability of generating a finite simple group. Geom. Dedic. 56, 103-113 (1995)

27. Malle, G.: The maximal subgroups of ${ }^{2} F_{4}\left(q^{2}\right)$. J. Algebra 139, 53-69 (1991)

28. O'Reilly Regueiro, E.: On primitivity and reduction for flag-transitive symmetric designs. J. Comb. Theory Ser. A 109, 135-148 (2005)

29. O'Reilly Regueiro, E.: Biplanes with flag-transitive automorphism groups of almost simple type, with alternating or sporadic socle. Eur. J. Comb. 26, 577-584 (2005)

30. O'Reilly-Regueiro, E.: Biplanes with flag-transitive automorphism groups of almost simple type, with classical socle. J. Algebr. Comb. (2007, to appear)

31. Salwach, C.J., Mezzaroba, J.A.: The four biplanes with $k=9$. J. Comb. Theory Ser. A 24, 141-145 (1978)

32. Saxl, J.: On finite linear spaces with almost simple flag-transitive automorphism groups. J. Comb. Theory Ser. A 100(2), 322-348 (2002)

33. Seitz, G.M.: Flag-transitive subgroups of Chevalley groups. Ann. Math. 97(1), 27-56 (1973)

34. Suzuki, M.: On a class of doubly transitive groups. Ann. Math. 75, 105-145 (1962) 